\title{
Wave-vector analysis of the jellium exchange-correlation surface energy in the random-phase approximation: Support for nonempirical density functionals
}

\author{
J. M. Pitarke, ${ }^{1,2}$ Lucian A. Constantin, ${ }^{3}$ and John P. Perdew ${ }^{3}$ \\ ${ }^{1}$ Materia Kondentsatuaren Fisika Saila, Zientzi Fakultatea, Euskal Herriko Unibertsitatea, 644 Posta kutxatila, E-48080 Bilbo, \\ Basque Country, Spain \\ ${ }^{2}$ Donostia International Physics Center (DIPC) and Unidad Física Materiales CSIC-UPV/EHU, Manuel de Lardizabal Pasealekua, \\ E-20018 Donostia, Basque Country, Spain \\ ${ }^{3}$ Department of Physics and Quantum Theory Group, Tulane University, New Orleans, Louisiana 70118, USA
}

(Received 11 May 2006; published 24 July 2006)

\begin{abstract}
We report a three-dimensional wave-vector analysis of the jellium exchange-correlation (xc) surface energy in the random-phase approximation (RPA). The RPA accurately describes long-range xc effects which are challenging for semilocal approximations, since it includes the universal small-wave-vector behavior derived by Langreth and Perdew. We use these rigorous RPA calculations for jellium slabs to test RPA versions of nonempirical semilocal density-functional approximations for the xc energy. The local spin density approximation displays canceling errors in the small- and intermediate-wave-vector regions. The Perdew-BurkeErnzerhof generalized gradient approximation improves the analysis for intermediate wave vectors, but remains too low for small wave vectors (implying too-low jellium xc surface energies). The nonempirical metageneralized gradient approximation of Tao, Perdew, Staroverov, and Scuseria gives a realistic wave-vector analysis, even for small wave vectors or long-range effects. We also study the effects of slab thickness and of short-range corrections to the RPA.
\end{abstract}

DOI: 10.1103/PhysRevB.74.045121

PACS number(s): 71.10.Ca, 71.15.Mb, 71.45.Gm

\section{INTRODUCTION}

Modern electronic-structure calculations for atoms, molecules, and solids usually rely upon Kohn-Sham (KS) density-functional theory (DFT), ${ }^{1,2}$ in which only $E_{\text {xc }}[n]$, the exchange-correlation (xc) energy as a functional of electron density, must be approximated. Semiempirical approximations tend to be limited to systems that resemble those in the fitted data set (typically small molecules), but nonempirical ones are constructed to satisfy universal constraints and so should have a wider range of applicability. ${ }^{3}$ For example, it is expected that a good description of chemical reactions at a solid surface requires a good description of both the molecules and the surface.

Jellium is a simple model of a simple metal, in which the valence electrons are neutralized by a uniform positive background that extends up to a sharp planar surface. The apparent success of the simplest density functional, the local spin density approximation (LSDA), for the jellium surface energy ${ }^{4}$ motivated early interest in density functionals and in refinements of the LSDA such as the PerdewBurke-Ernzerhof (PBE) generalized gradient approximation (GGA). 5,6

It was therefore a matter of some concern when wavefunction-based Fermi hypernetted-chain ${ }^{7}$ and fixed-node diffusion Monte $\mathrm{Carlo}^{8}$ (DMC) calculations for jellium slabs (and their extrapolation to infinite thickness) predicted surface energies considerably higher than those obtained in the LSDA. Indeed, the DMC method is usually a gold standard of accuracy. However, it encounters special difficulties for jellium slabs; ${ }^{9}$ furthermore, the large deviations between the available DMC and LSDA calculations have been attributed in part to inconsistency between the energy of the inhomogeneous system and that of the corresponding homogeneous electron gas. ${ }^{10,11}$ Recent approaches ${ }^{10-16}$ have all suggested that the actual jellium surface energies are only a little higher than those obtained in the LSDA. The jellium surface-energy story is presented in full detail in Ref. 16.

In this paper, we perform a detailed analysis of exchange and correlation in jellium slabs, exact at the level of the random-phase approximation (RPA), to show that the most refined nonempirical density functional, the metageneralized gradient approximation of Tao, Perdew, Staroverov, and Scuseria (TPSS meta-GGA), ${ }^{17}$ can account even for the most long-ranged xc effects at a jellium surface. This is a considerable achievement for a semilocal functional that is inherently more reliable for short-ranged effects than for longranged ones. The RPA is known to be correct at long range; because it has serious deficiencies at short range and, therefore, cannot be compared to standard versions of the semilocal functionals, we use RPA versions of these functionals in this test.

In order to separate long-range and short-range xc effects, we look at the surface contribution to the spherically averaged real-space xc hole, averaged over the electron density of the system, and its Fourier transform (wave-vector analysis). Langreth and Perdew ${ }^{5}$ showed that the exact xc energy of an arbitrary inhomogeneous system can be obtained from a three-dimensional (3D) Fourier transform of the spherical average of the $\mathrm{xc}$ hole density, which is a function of a $3 \mathrm{D}$ wave vector $\mathbf{k}$. In the case of a plane-bounded electron gas, this wave-vector-dependent spherical average is dominated at long wavelengths $(k \rightarrow 0)$ by the zero-point energy shift of the newly created surface collective oscillations (surface plasmons) and takes a simple analytical form. This known limit has been used to carry out a wave-vector interpolation correction to the LSDA, ${ }^{5} \mathrm{PBE}$ GGA, ${ }^{13}$ and TPSS-metaGGA (Ref. 16) xc surface energies. The wave-vector interpolation 
corrections to these functionals were controlled ${ }^{13,16}$ by using the exact RPA values reported in Ref. 12, and led to a consistent set of predicted surface energies. ${ }^{16}$

In a DFT context, the RPA is based upon the timedependent Hartree approximation for the density-response function but replacing the occupied and unoccupied singleparticle Hartree orbitals and energies by the corresponding eigenfunctions and eigenvalues of the KS Hamiltonian of DFT. ${ }^{5}$ Hence, it describes the exchange energy and the longrange part of the correlation energy correctly. Essentially exact RPA surface energies were evaluated from single-particle LSDA orbitals and energies in Ref. 12. These calculations provide an accurate standard against which approximate density functionals (in their RPA versions) can be tested and normed. The RPA versions of the LSDA and GGA were reported in Refs. 18 and 19, respectively. Because the RPA is not self-correlation-free, the GGA for RPA correlation is its own meta-GGA. The RPA version of the nonempirical TPSS meta-GGA was investigated in Ref. 16.

Unless stated otherwise, atomic units are used throughout, i.e., $e^{2}=\hbar=m_{e}=1$.

\section{THEORETICAL FRAMEWORK}

The exact xc energy $E_{\mathrm{xc}}[n]$ of an arbitrary inhomogeneous system of density $n(\mathbf{r})$ can be obtained from the spherical average $\bar{n}_{\mathrm{xc}}(\mathbf{r}, u)$ of the coupling-constant-averaged xc hole density $\bar{n}_{\mathrm{xc}}\left(\mathbf{r}, \mathbf{r}^{\prime}\right)$ at $\mathbf{r}^{\prime}$ around an electron at $\mathbf{r}$, as follows: ${ }^{5,16}$

$$
E_{\mathrm{xc}}[n]=\int d \mathbf{r} n(\mathbf{r}) \varepsilon_{\mathrm{xc}}[n](\mathbf{r}),
$$

where $\varepsilon[n](\mathbf{r})$ represents the xc energy per particle at point $\mathbf{r}$ :

$$
\varepsilon_{\mathrm{xc}}[n](\mathbf{r})=4 \int_{0}^{\infty} d k \int_{0}^{\infty} d u u^{2} \frac{\sin k u}{k u} \bar{n}_{\mathrm{xc}}(\mathbf{r}, u),
$$

with

$$
\bar{n}_{\mathrm{xc}}(\mathbf{r}, u)=\frac{1}{4 \pi} \int d \Omega \bar{n}_{\mathrm{xc}}\left(\mathbf{r}, \mathbf{r}^{\prime}\right),
$$

$d \Omega$ being a differential solid angle around the direction of $\mathbf{u}=\mathbf{r}^{\prime}-\mathbf{r}$.

The xc surface energy $\sigma_{\mathrm{xc}}$ is obtained by subtracting from the xc energy $E_{\mathrm{xc}}[n]$ of a semi-infinite electron system the corresponding energy $E_{\mathrm{xc}}^{\text {unif }}(n)$ of a uniform electron gas. In a jellium model, in which the electron system is translationally invariant in the plane of the surface, and assuming the surface to be normal to the $z$ axis, one finds

$$
\sigma_{\mathrm{xc}}=\int_{0}^{\infty} d\left(\frac{k}{2 k_{F}}\right) \gamma_{\mathrm{xc}}(k)
$$

where $^{20}$

$$
\gamma_{\mathrm{xc}}(k)=2 \frac{k_{F}}{\pi} \int_{-\infty}^{+\infty} d z n(z) b_{\mathrm{xc}}(k, z),
$$

with $k_{F}=\left(3 \pi^{2} \bar{n}\right)^{1 / 3}, \bar{n}$ being the background density, and

$$
b_{\mathrm{xc}}(k, z)=4 \pi \int_{0}^{\infty} d u u^{2} \frac{\sin k u}{k u}\left[\bar{n}_{\mathrm{xc}}(z, u)-\bar{n}_{\mathrm{xc}}^{\mathrm{unif}}(u)\right] .
$$

Alternatively, one can introduce Eq. (3) into Eq. (6) to find

$$
b_{\mathrm{xc}}(k, z)=\frac{1}{2} \int_{-k}^{+k} \frac{d k_{z}}{k} \int_{-\infty}^{+\infty} d z^{\prime} e^{i k_{z}\left(z-z^{\prime}\right)} \bar{n}_{\mathrm{xc}}\left(k_{\|} ; z, z^{\prime}\right)-\bar{n}_{\mathrm{xc}}^{\mathrm{unif}}(k),
$$

with $k_{\|}=\sqrt{k^{2}-k_{z}^{2}}$, and $\bar{n}_{\mathrm{xc}}\left(k_{\|} ; z, z^{\prime}\right)$ and $\bar{n}_{\mathrm{xc}}^{\mathrm{unif}}(k)$ representing Fourier transforms of the coupling-constant-averaged xc hole densities $\bar{n}_{\mathrm{xc}}\left(\mathbf{r}, \mathbf{r}^{\prime}\right)$ and $\bar{n}_{\mathrm{xc}}^{\text {unif }}\left(\mathbf{r}, \mathbf{r}^{\prime}\right)$, respectively. At long wavelengths $(k \rightarrow 0)$, one finds the exact limit ${ }^{5}$

$$
\gamma_{\mathrm{xc}}(k)=\frac{k_{F}}{4 \pi}\left(\omega_{s}-\frac{1}{2} \omega_{p}\right) k,
$$

which only depends on the bulk- and surface-plasmon energies $\omega_{p}=(4 \pi \bar{n})^{1 / 2}$ and $\omega_{s}=\omega_{p} / \sqrt{2}$, and does not depend, therefore, on the electron-density profile at the surface.

The spherical average $\bar{n}_{\mathrm{xc}}(z, u)$ entering Eq. (6) can be obtained within local or semilocal density-functional approximations (such as LSDA, PBE GGA, and TPSS metaGGA) from models ${ }^{16,19,21,22}$ that require knowledge of the $x c$ hole density $\bar{n}_{\mathrm{xc}}^{\mathrm{unif}}(u)$ of a uniform electron gas. Alternatively, rigorous calculations of $\bar{n}_{\mathrm{xc}}^{\mathrm{unif}}(k)$ and the fully nonlocal $\bar{n}_{\mathrm{xc}}\left(k_{\|} ; z, z^{\prime}\right)$ entering Eq. (7) can be carried out from knowledge of the $\lambda$-dependent density-response functions $\chi_{\text {unif }}^{\lambda}(k, \omega)$ and $\chi^{\lambda}\left(k_{\|} \omega ; z, z^{\prime}\right)$, respectively, defined by adiabatically switching on the $e$-e interaction via the coupling constant $\lambda$ and by adding, at the same time, an external potential so as to maintain the true $(\lambda=1)$ ground-state density in the presence of the modified $e$-e interaction. ${ }^{23,24} \mathrm{By}$ using the fluctuation-dissipation theorem, ${ }^{25,26}$ one finds

$$
\bar{n}_{\mathrm{xc}}^{\mathrm{unif}}(k)=\frac{1}{\bar{n}}\left(-\frac{1}{\pi} \int_{0}^{1} d \lambda \int_{0}^{\infty} d \omega \chi_{\mathrm{unif}}^{\lambda}(k, i \omega)-\bar{n}\right)
$$

and

$$
\begin{aligned}
\bar{n}_{\mathrm{xc}}\left(k_{\|} ; z, z^{\prime}\right)= & -\frac{1}{\pi n(z)} \int_{0}^{1} d \lambda \int_{0}^{\infty} d \omega \chi^{\lambda}\left(k_{\|}, i \omega ; z, z^{\prime}\right) \\
& -\delta\left(z-z^{\prime}\right) .
\end{aligned}
$$

With the aim of testing the performance of local and semilocal density-functional approximations for the xc surface energy, we compare these (local and semilocal) calculations [obtained from Eq. (6)] to their fully nonlocal counterparts [obtained from Eq. (7) with the aid of Eqs. (9) and (10)] at the same level of approximation, which we choose to be the RPA. On the one hand, we evaluate $\gamma_{\mathrm{xc}}(k)$ from RPA versions (LSDA RPA, PBE RPA, and TPSS RPA) of the local (or semilocal) $\bar{n}_{\mathrm{xc}}(z, u)$ entering Eq. (6) based on the RPA xc hole density $\bar{n}_{\mathrm{xc}}^{\text {unif }}(u)$ of a uniform electron gas. On the other hand, we evaluate $\gamma_{\mathrm{xc}}(k)$ from a fully nonlocal version (exact RPA) of $\bar{n}_{\mathrm{xc}}\left(k_{\|} ; z, z^{\prime}\right)$ entering Eq. (7) based [by using Eq. (10)] on the RPA density-response function $\chi^{\lambda}\left(k_{\|}, \omega ; z, z^{\prime}\right)$. 


\section{RESULTS}

In the calculations presented below, we have considered a jellium slab of background thickness $a=2.23 \lambda_{F}, \lambda_{F}$ being the Fermi wavelength $\left(\lambda_{F}=2 \pi / k_{F}\right)$, and background density $\bar{n}$ $=\left[(4 \pi / 3) r_{s}^{3}\right]^{-1}$ with $r_{s}=2.07$. This slab corresponds to about four atomic layers of $\mathrm{Al}(100)$.

For the LSDA RPA calculations, we have obtained the RPA xc hole density $\bar{n}_{\mathrm{xc}}^{\text {unif }}(u)$ of a uniform electron gas either from Eq. (9) or from a nonoscillatory parametrization. ${ }^{27}$ For the PBE RPA and TPSS RPA calculations, we have always used a nonoscillatory parametrization of the RPA xc hole density $\bar{n}_{\mathrm{xc}}^{\text {unif }}(u) .^{27}$

For the evaluation of the fully nonlocal (exact RPA) $\gamma_{\mathrm{xc}}(k)$ of Eq. (5), we follow the method described in Ref. 12. We first assume that $n(z)$ vanishes at a distance $z_{0}$ from either jellium edge, ${ }^{28}$ and we expand the single-particle wave functions $\phi_{l}(z)$ and the density-response function $\chi^{\lambda}\left(k_{\|}, \omega ; z, z^{\prime}\right)$ in sine and double-cosine Fourier representations, respectively. We then perform the integrals over the coordinates $z$ and $z^{\prime}$ analytically, and we find an explicit expression for $\gamma_{\mathrm{xc}}(k)$ [see Eqs. (A1)-(A5) of the Appendix] in terms of the single-particle energies $\varepsilon_{l}$ and the Fourier coefficients $b_{l s}$ and $\chi_{m n}\left(k_{\|}, \omega\right)$ of the single-particle wave functions $\phi_{l}(z)$ and the density-response function $\chi^{\lambda}\left(k_{\|}, \omega ; z, z^{\prime}\right)$, respectively. ${ }^{29} \mathrm{We}$ have taken all the single-particle wave functions $\phi_{l}(z)$ and energies $\varepsilon_{l}$ to be the LDA eigenfunctions and eigenvalues of the KS Hamiltonian of DFT, as obtained by using the Perdew-Wang parametrization ${ }^{18}$ of the Ceperley-Alder $\mathrm{xc}$ energy of the homogeneous electron gas. ${ }^{30}$ For the jellium slab with $r_{s}=2.07$ and $a=2.23 \lambda_{F}$ considered here, the exact RPA xc surface energy is found to be $\sigma_{\mathrm{xc}}=3091 \mathrm{erg} / \mathrm{cm}^{2}$, not far from the corresponding RPA xc surface energy of a semiinfinite jellium which is known to be $\sigma_{\mathrm{xc}}=3064 \mathrm{erg} / \mathrm{cm}^{2} .{ }^{10}$

In Figs. 1 and 2 we have plotted (solid lines) the exact exchange contribution to $\gamma_{\mathrm{xc}}(k)$, i.e., $\gamma_{x}(k)$, which we have obtained from Eqs. (A1)-(A5) with the quantities $\chi_{\text {unif }}^{\lambda}(k, \omega)$ and $\chi_{m n}^{\lambda}\left(k_{\|} \omega\right)$ replaced by their noninteracting counterparts $\chi_{\text {unif }}^{0}(k, \omega)$ and $\chi_{m n}^{0}\left(k_{\|}, \omega\right)$, respectively. Also plotted in these figures are the LSDA, PBE, and TPSS calculations of $\gamma_{x}(k)$ that we have obtained by replacing the xc hole densities $\bar{n}_{\mathrm{xc}}(z, u)$ and $\bar{n}_{\mathrm{xc}}^{\text {unif }}(u)$ entering Eq. (6) by their corresponding exchange-only counterparts (dashed lines).

The LSDA $\gamma_{x}(k)$ represented in Fig. 1 has been obtained by using both the actual exchange hole density $n_{x}^{\text {unif }}(u)$ of a uniform electron gas (dashed curve labeled $\gamma_{x}^{\mathrm{LSDA}}$ ), which we have obtained from Eq. (9) with $\chi_{\text {unif }}^{\lambda}(k, \omega)$ replaced by $\chi_{\text {unif }}^{0}(k, \omega)$, and the nonoscillatory exchange hole density $n_{x}^{\text {unif }}(u)$ reported in Ref. 22 (dashed curve labeled $\langle\gamma\rangle_{x}^{\mathrm{LSDA}}$ ). $\gamma_{x}^{\mathrm{LSDA}}(k)$ and $\langle\gamma\rangle_{x}^{\mathrm{LSDA}}(k)$ yield, by construction of the nonoscillatory exchange hole density $n_{x}^{\text {unif }}(u)$, the same exchange surface energy $\sigma_{x}$; they are also almost identical in a wide range of low wave vectors, but $\langle\gamma\rangle_{x}^{\mathrm{LSDA}}(k)$ is considerably less accurate near $k=2 k_{F}$ where the exact $\gamma_{x}(k)$ has a kink. This kink is realistic for jellium-like systems, but not for atoms and molecules.

The PBE and TPSS $\gamma_{x}(k)$ represented in Fig. 2 have both been obtained by using the nonoscillatory exchange hole

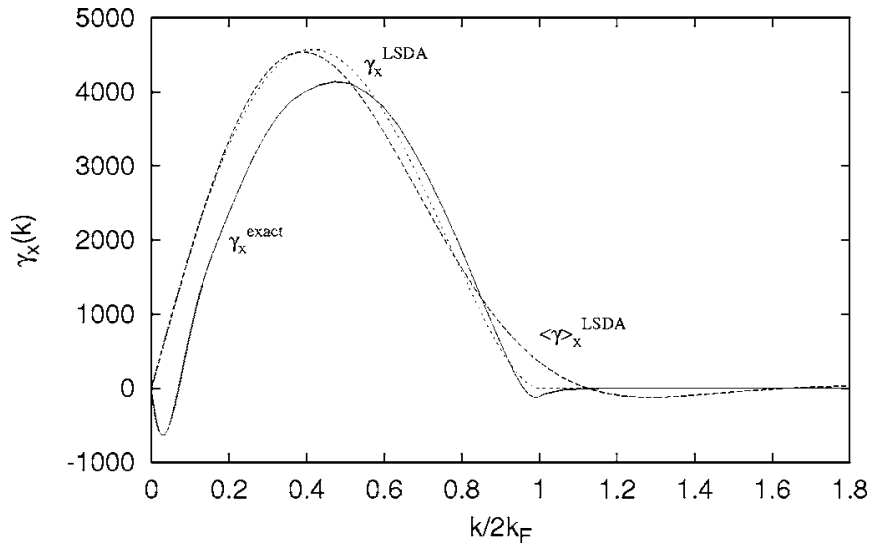

FIG. 1. Wave-vector analysis $\gamma_{x}(k)$, versus $k / 2 k_{F}$, of the exchange surface energy of a jellium slab of thickness $a=2.23 \lambda_{F}$ and $r_{s}=2.07$. Solid and dashed lines represent exact and LSDA calculations, respectively. The LSDA calculation has been performed either from the actual exchange hole density $n_{x}^{\text {unif }}(u)$ of a uniform electron gas, which we have obtained from Eq. (9) with $\chi_{\text {unif }}^{\lambda}(k, \omega)$ replaced by $\chi_{\text {unif }}^{0}(k \omega)\left(\gamma_{x}^{\mathrm{LSDA}}\right)$ or from the nonoscillatory parametrization of $n_{x}^{\text {unif }}(u)$ reported in Ref. $22\left(\langle\gamma\rangle_{x}^{\mathrm{LSDA}}\right)$. The area under each curve represents the exchange surface energy: $\sigma_{x}^{\mathrm{LSDA}}=2699 \mathrm{erg} / \mathrm{cm}^{2}$ and $\sigma_{x}^{\text {exact }}=2348 \mathrm{erg} / \mathrm{cm}^{2}$. $\left(1\right.$ hartree $/ \mathrm{bohr}^{2}$ $=1.557 \times 10^{6} \mathrm{erg} / \mathrm{cm}^{2}$.)

density $n_{x}^{\text {unif }}(u)$ reported in Ref. 22, which yields a wrong behavior of $\gamma_{x}(k)$ at large wave vectors. Nevertheless, both the actual exchange hole density $n_{x}^{\text {unif }}(u)$ of a uniform electron gas (not used in these calculations) and the corresponding nonoscillatory exchange hole density would yield the same exchange surface energy $\sigma_{x}$, by construction, as occurs in the LSDA.

Figures 1 and 2 show that while the LSDA $\gamma_{x}(k)$ considerably overestimates the exact $\gamma_{x}(k)$ at low wave vectors (see Fig. 1), leading to an exchange surface energy $\sigma_{x}$ that is too

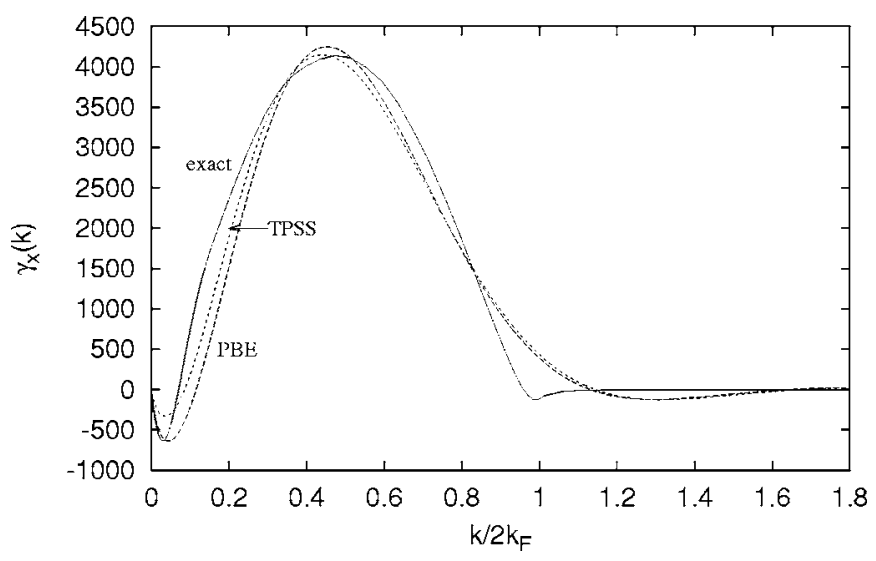

FIG. 2. Wave-vector analysis $\gamma_{x}(k)$, versus $k / 2 k_{F}$, of the exchange surface energy of a jellium slab of thickness $a=2.23 \lambda_{F}$ and $r_{s}=2.07$. Solid and dashed lines represent exact and semilocal (PBE and TPSS) calculations, respectively. The semilocal PBE and TPSS calculations have been performed from the nonoscillatory parametrization of $n_{x}^{\text {unif }}(u)$ reported in Ref. 22. The area under each curve represents the exchange surface energy: $\sigma_{x}^{\mathrm{PBE}}=2155 \mathrm{erg} / \mathrm{cm}^{2}$, $\sigma_{x}^{\mathrm{TPSS}}=2247 \mathrm{erg} / \mathrm{cm}^{2}$, and $\sigma_{x}^{\text {exact }}=2348 \mathrm{erg} / \mathrm{cm}^{2}$. 


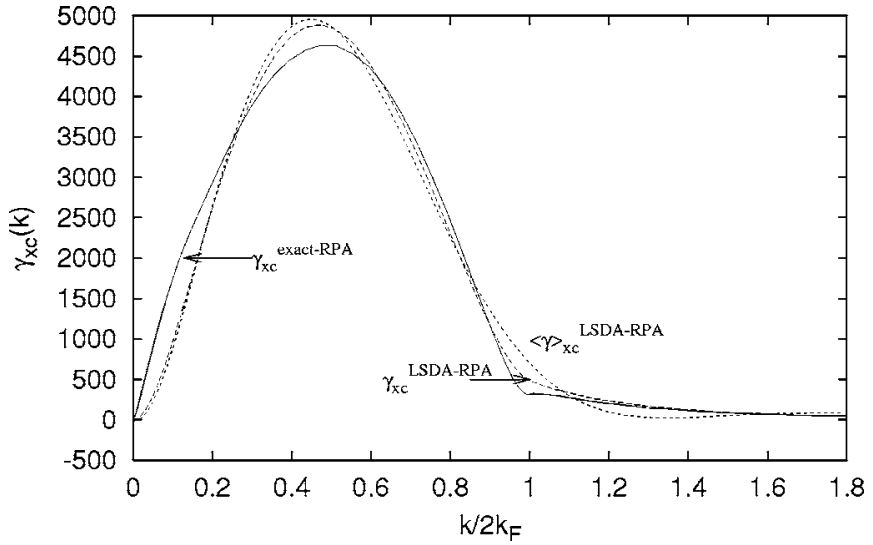

FIG. 3. Wave-vector analysis $\gamma_{\mathrm{xc}}(k)$, versus $k / 2 k_{F}$, of the RPA xc surface energy of a jellium slab of thickness $a=2.23 \lambda_{F}$ and $r_{s}=2.07$. Solid and dashed lines represent exact RPA and LSDA RPA calculations, respectively. The LSDA calculation has been performed either from the actual RPA xc hole density of Eq. (9) $\left(\gamma_{\mathrm{xc}}^{\mathrm{LSDARPA}}\right)$ or from a nonoscillatory parametrization of $\bar{n}_{\mathrm{xc}}^{\text {unif }}(u)$ $\left.(\langle\gamma\rangle\rangle_{\mathrm{xc}}^{\mathrm{LSDA}}\right) .{ }^{27}$ The area under each curve represents the RPA xc surface energy: $\sigma_{\text {xc }}^{\text {LSDA RPA }}=3034 \mathrm{erg} / \mathrm{cm}^{2}$ and $\sigma_{\text {xc }}^{\text {exact RPA }}$ $=3091 \mathrm{erg} / \mathrm{cm}^{2}$.

large, the PBE and TPSS $\gamma_{x}(k)$ are close to the exact $\gamma_{x}(k)$ (see Fig. 2). We note that the peaks of $\gamma_{x}^{\mathrm{PBE}}(k)$ and $\gamma_{x}^{\mathrm{TPSS}}(k)$ are close to the exact one, a fact which was used in the construction of the TPSS exchange hole, ${ }^{16}$ and that at larger wave vectors $\gamma_{x}^{\mathrm{PBE}}(k)$ and $\gamma_{x}^{\mathrm{TPSS}}(k)$ nearly coincide, as expected; at lower wave vectors, however, the TPSS metaGGA differs from the PBE GGA, leading to a wave-vectordependent $\gamma_{x}(k)$ that is closer to the exact behavior.

We have also carried out calculations of the exact $\gamma_{x}(k)$ for increasing values of the background thickness $a$, and we have found that (i) $\gamma_{x}(k)$ is sensitive to the size of the system only at wave vectors below the minimum that is present in the solid lines of Figs. 1 and 2, and (ii) as $k \rightarrow 0$ the wave-vector-dependent $\gamma_{x}(k)$ approaches in the semi-infinite limit the profile-independent negative value $\left(\gamma_{x}=-1.50 \times 10^{4} / r_{s}^{3} \mathrm{erg} / \mathrm{cm}^{2}\right)$ reported in Refs. 5 and 31 .

Figures 3 and 4 exhibit the results that we have obtained for the RPA $\gamma_{\mathrm{xc}}(k)$ from Eqs. (A1)-(A5) (solid lines) and within the LSDA RPA, PBE RPA, and TPSS RPA (dashed lines). As in the case of the exchange-only contributions represented in Figs. 1 and 2, the LSDA $\gamma_{\mathrm{xc}}(k)$ represented in Fig. 3 has been obtained by using both the actual RPA xc hole density $\bar{n}_{\mathrm{xc}}^{\text {unif }}(u)$ (dashed line labeled $\left.\gamma_{\mathrm{xc}}^{\mathrm{LSDA} R P A}\right)$, which we have obtained from Eq. (9), and a nonoscillatory xc hole density $\bar{n}_{\mathrm{xc}}^{\text {unif }}(u)$ (dashed line labeled $\langle\gamma\rangle_{\mathrm{xc}}^{\mathrm{LSDA} R P A}$ ); the PBE and TPSS $\gamma_{\mathrm{xc}}(k)$ represented in Fig. 4 have both been obtained by using a nonoscillatory xc hole density $\bar{n}_{\mathrm{xc}}^{\mathrm{unif}}(u)$.

Figure 3 shows that at short wavelengths with $k>2 k_{F}$ the quantities $\gamma_{\mathrm{xc}}^{\mathrm{LSDA} R P A}(k)$ (dashed line) and $\gamma_{\mathrm{xc}}^{\text {exact RPA }}(k)$ (solid line) nearly coincide, as expected. ${ }^{5,32,33}$ The LSDA, however, considerably underestimates $\gamma_{\mathrm{xc}}(k)$ at low wave vectors. This is partially compensated by a LSDA $\gamma_{\mathrm{xc}}(k)$ that at intermediate wave vectors [around the peak of $\gamma_{\mathrm{xc}}(k)$ ] is too large. Figure 4 shows that the PBE GGA improves $\gamma_{\mathrm{xc}}(k)$ at inter-

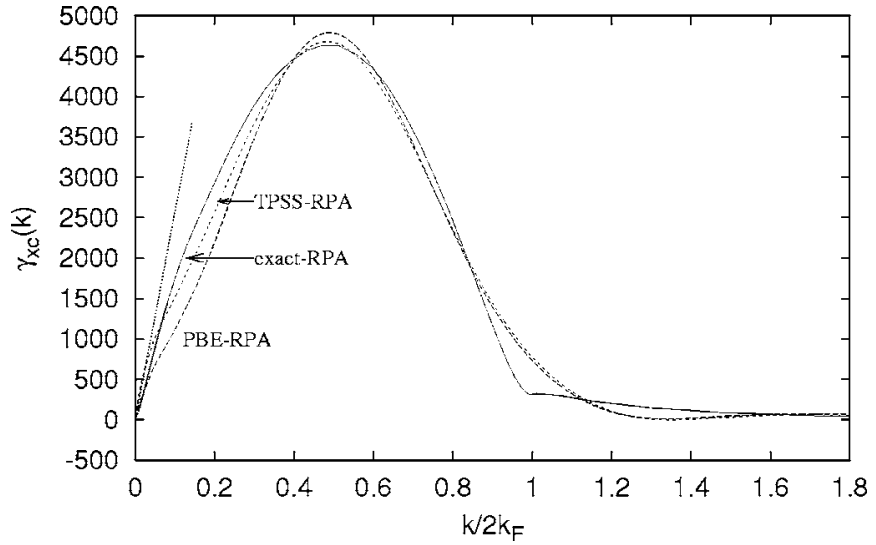

FIG. 4. Wave-vector analysis $\gamma_{\mathrm{xc}}(k)$, versus $k / 2 k_{F}$, of the RPA xc surface energy of a jellium slab of thickness $a=2.23 \lambda_{F}$ and $r_{s}=2.07$. Solid and dashed lines represent exact RPA and semilocal RPA (PBE RPA and TPSS RPA) calculations, respectively. The semilocal PBE RPA and TPSS RPA calculations have been performed from a nonoscillatory parametrization of $\bar{n}_{\mathrm{xc}}^{\text {unif }}(u)$ (Ref. 27). The area under each curve represents the RPA xc surface energy: $\sigma_{\mathrm{xc}}^{\mathrm{PBE} R P A}=2959 \mathrm{erg} / \mathrm{cm}^{2}, \quad \sigma_{\mathrm{xc}}^{\mathrm{TPSS} \mathrm{RPA}}=3052 \mathrm{erg} / \mathrm{cm}^{2}, \quad$ and $\sigma_{\text {xc }}^{\text {exact } \mathrm{RPA}^{\mathrm{xc}}}=3091 \mathrm{erg} / \mathrm{cm}^{2}$. The straight dotted line represents the universal low-wave-vector limit of Eq. (8).

mediate wave vectors more than at low wave vectors, thereby yielding an xc surface energy that is even smaller than in the LSDA. From a different perspective, ${ }^{34}$ the too-small PBE surface energy arises from a too-large gradient coefficient for exchange, but this is repaired by the TPSS meta-GGA which uses the proper gradient coefficient. Indeed, Fig. 4 clearly shows that the TPSS metaGGA brings improvements over the corresponding PBE GGA at both intermediate and small wave vectors, thus leading to a wave-vector-dependent $\gamma_{\mathrm{xc}}^{\mathrm{TPSS} \operatorname{RPA}}(k)$ that is very close to $\gamma_{\mathrm{xc}}^{\text {exact } \mathrm{RPA}}(k)$ (solid line) and to an xc surface energy $\sigma_{\mathrm{xc}}$ that is only slightly lower than its exact RPA counterpart. ${ }^{35}$ We have obtained similar results (not displayed here) for $r_{s}=3$, and we have found that the errors introduced by the use of nonempirical semilocal densityfunctional approximations slightly increase with $r_{s}$ as expected from the analysis of Ref. 16.

Also represented in Fig. 4 (by a dotted line) is the universal (density-profile-independent) low-wave-vector limit of Eq. (8). The TPSS RPA $\gamma_{\mathrm{xc}}(k)$ has the virtue that not only is it very close to its exact RPA counterpart in the whole range of low and intermediate wave vectors, but it imitates the exact low-wave-vector limit of Eq. (8) as well. That this limit is also reproduced by the exact RPA $\gamma_{\mathrm{xc}}(k)$ of a semi-infinite electron system is shown in Fig. 5, where we have plotted calculations of this quantity for increasing values of the background thickness $a$, from $a=0.56 \lambda_{F}$ to $8.23 \lambda_{F}$. Furthermore, Fig. 6 shows that $\gamma_{\mathrm{xc}}(k)$ is only sensitive to the background thickness at very low wave vectors.

Finally, in order to investigate the impact of short-range corrections to the RPA $\gamma_{\mathrm{xc}}(k)$, we have plotted in Fig. 7 the correlation contribution to $\gamma_{\mathrm{xc}}(k)$, i.e., $\gamma_{c}(k)$, as obtained in the RPA (LSDA RPA, TPSS RPA, and exact RPA) and also in standard versions of local and semilocal density function- 


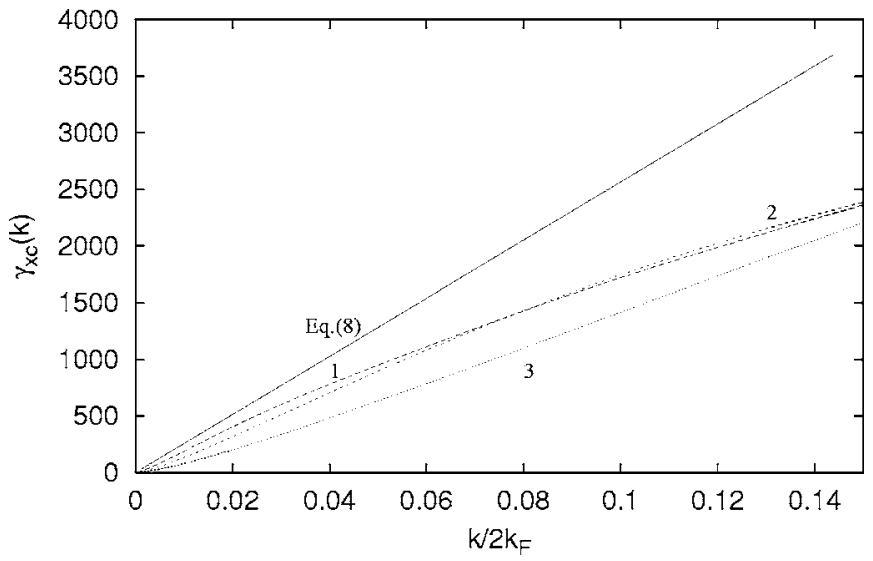

FIG. 5. Wave-vector analysis $\gamma_{\mathrm{xc}}(k)$, versus $k / 2 k_{F}$, of the exact RPA xc surface energy of jellium slabs of $r_{s}=2.07$ and various values of the background thickness: $a=8.23 \lambda_{F}(1), 2.23 \lambda_{F}(2)$, and $0.56 \lambda_{F}(3)$. The straight solid line represents the universal lowwave-vector limit of Eq. (8), which corresponds to a plane-bonded semi-infinite system $(a \rightarrow \infty)$.

als (LSDA and TPSS) that use an accurate (beyond RPA) nonoscillatory parametrization of the correlation hole density $\bar{n}_{c}^{\text {unif }}(u)$ of a uniform electron gas. ${ }^{21}$ We observe that in the long-wavelength limit $(k \rightarrow 0)$, where both the LSDA RPA and standard LSDA exhibit serious deficiencies, both the TPSS RPA and the more accurate standard TPSS results coincide with the exact RPA. At shorter wavelengths, the standard TPSS method predicts a substantial correction to its TPSS RPA and exact RPA counterparts, which is first positive and then negative and leads, therefore, to a persistent cancellation of short-range correlation effects beyond the RPA similar to the cancellation that was reported in Ref. 10 in the framework of time-dependent density-functional theory and a two-dimensional wave-vector analysis of the correlation surface energy.

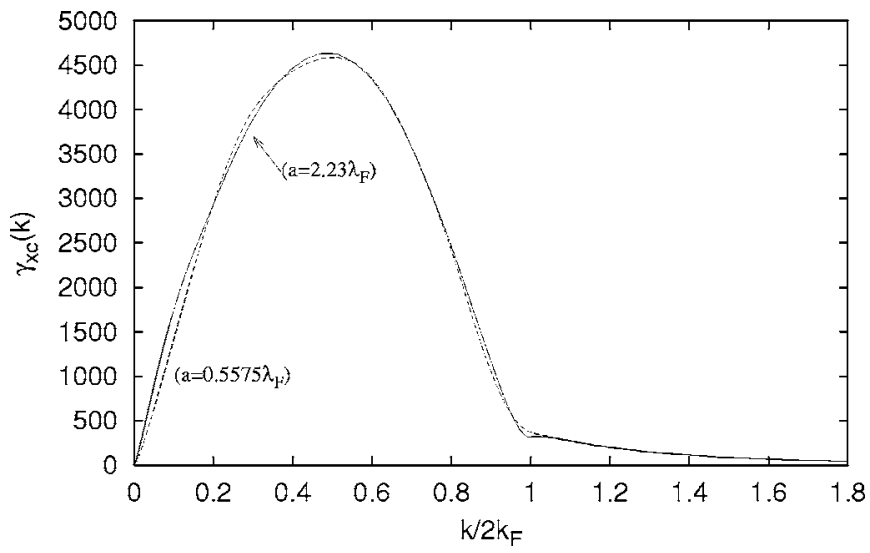

FIG. 6. Wave-vector analysis $\gamma_{\mathrm{xc}}(k)$, versus $k / 2 k_{F}$, of the exact RPA xc surface energy of jellium slabs of $r_{s}=2.07$ and two values of the background thickness: $a=2.23 \lambda_{F}$ (solid line) and $0.56 \lambda_{F}$ (dashed line). The area under each curve represents the exact RPA

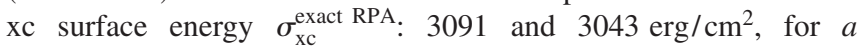
$=2.23 \lambda_{F}$ and $0.56 \lambda_{F}$, respectively.

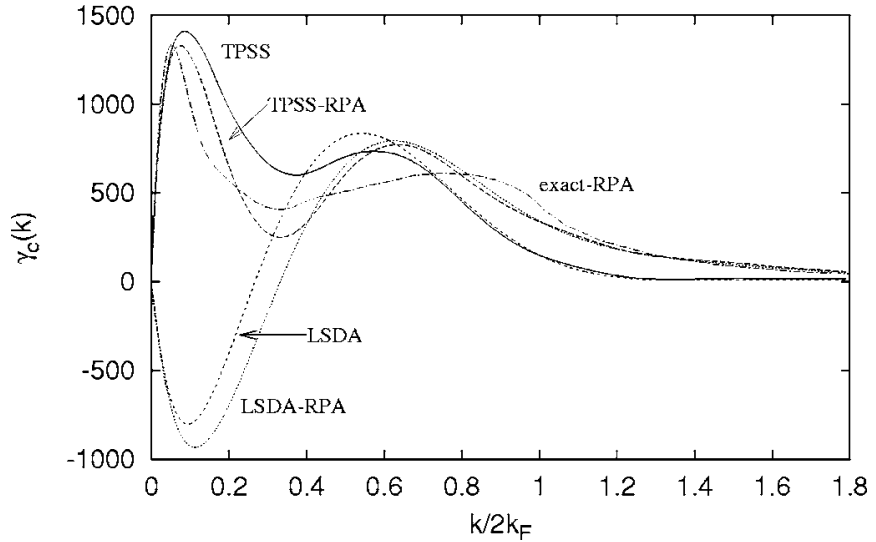

FIG. 7. Wave-vector analysis $\gamma_{c}(k)$, versus $k / 2 k_{F}$, of the correlation surface energy of a jellium slab of thickness $a=2.23 \lambda_{F}$ and $r_{s}=2.07$. Dotted, long-dashed, and dash-dotted lines represent LSDA RPA, TPSS RPA, and exact RPA calculations, respectively. Short-dashed and solid lines represent standard versions of the LSDA and the semilocal TPSS, as obtained from an accurate (beyond RPA) nonoscillatory parametrization of the correlation hole density $\bar{n}_{c}^{\text {unif }}(u)$ of a uniform electron gas (Ref. 21). The area under each curve represents the correlation surface energy: $\sigma_{c}^{\text {LSDA RPA }}$ $=336 \mathrm{erg} / \mathrm{cm}^{2}, \sigma_{c}^{\text {TPSS RPA }}=804 \mathrm{erg} / \mathrm{cm}^{2}, \sigma_{c}^{\text {exact RPA }}=743 \mathrm{erg} / \mathrm{cm}^{2}$, $\sigma_{c}^{\mathrm{LSDA}}=290 \mathrm{erg} / \mathrm{cm}^{2}$, and $\sigma_{c}^{\mathrm{TPSS}}=756 \mathrm{erg} / \mathrm{cm}^{2}$.

\section{CONCLUSIONS}

We have reported the first 3D wave-vector analysis of the jellium xc surface energy in the RPA, and we have used this fully nonlocal (essentially exact) RPA calculation to test RPA versions of nonempirical semilocal density-functional approximations for the xc energy. We have tested the first three rungs of the Jacob's ladder classification of nonempirical density functionals: ${ }^{36}$ the LSDA, PBE GGA, and TPSS meta-GGA.

We have found that while the LSDA displays canceling errors in the small- and intermediate-wave-vector regions and the PBE GGA improves the analysis for intermediate wave vectors while remaining too low for small wave vectors (implying two-low xc surface energies), the TPSS metaGGA yields a realistic wave-vector analysis even for small wave vectors or long-range effects. We have also demonstrated numerically the correctness of the LSDA at large wave vectors ${ }^{5,32,33}$ (where the LSD RPA, TPSS RPA, and exact RPA coincide, as shown in Fig. 7) and the universal low-wave-vector behavior derived by Langreth and Perdew, ${ }^{5}$ which is nicely reproduced by the TPSS meta-GGA.

We have carried out fully nonlocal RPA calculations for increasing values of the background thickness, and we have found that the 3D wave-vector analysis of the xc surface energy is remarkably insensitive to the slab thickness except at very long wavelengths $(k \rightarrow 0)$ where decreasing the slab thickness reduces the universal slope that is dictated by the presence of bulk and surface collective oscillations.

Finally, we have found that the TPSS wave-vector analysis of the correlation surface energy, as obtained from an accurate (beyond RPA) nonoscillatory parametrization of the 
xc hole density of a uniform electron gas, provides both the exact short- $k$ limit, where the LDA fails badly, and the exact large- $k$ limit, where the RPA is wrong. Hence, our calculations support the conclusion that the TPSS meta-GGA xc density functional accurately describes the jellium surface, including not only short-range but also long-range effects.

\section{ACKNOWLEDGMENTS}

J.M.P. acknowledges partial support by the University of the Basque Country, the Basque Unibertsitate eta Ikerketa Saila, the Spanish Ministerio de Educación y Ciencia, and the EC 6th framework Network of Excellence NANOQUANTA (Grant No. NMP4-CT-2004-500198). L.A.C. and J.P.P. acknowledge the support of the U.S. National Science Foundation under Grant No. DMR-0501588.

\section{APPENDIX}

Here we give an explicit expression for the wave-vectordependent contribution $\gamma_{\mathrm{xc}}(k)$ to the xc surface energy $\sigma_{\mathrm{xc}}$ of a jellium slab of background density $\bar{n}$ and thickness $a$, in terms of the single-particle energies $\varepsilon_{l}$ and the Fourier coefficients $b_{l s}$ and $\chi_{m n}^{\lambda}$ of the single-particle wave functions $\phi_{l}(z)$ and the density-response function $\chi_{\lambda}\left(k_{\|} ; z, z^{\prime}\right)$, respectively. ${ }^{29}$ From Eqs. (5), (7), (9), and (10) and performing the integrals over the coordinates $z$ and $z^{\prime}$ analytically, we find

$$
\gamma_{\mathrm{xc}}(k)=\frac{k_{F}}{\pi}\left(\int_{0}^{k} \frac{d k_{z}}{k} \sum_{m=0}^{\infty} \sum_{n=0}^{\infty} \alpha_{m n}\left(k_{z}\right) \beta_{m n}\left(k_{\|}\right)-\bar{n} a \bar{n}_{\mathrm{xc}}^{\mathrm{unif}}(k)\right),
$$

where

$$
\alpha_{m n}\left(k_{z}\right)=2 k_{z}^{2} \frac{1-(-1)^{m} \cos \left(k_{z} d\right)}{\left[k_{z}^{2}-(m \pi / d)^{2}\right]\left[k_{z}^{2}-(n \pi / d)^{2}\right]}
$$

and $^{12}$

$$
\begin{aligned}
\beta_{m n}\left(k_{\|}\right)= & -\frac{1}{\pi} \int_{0}^{1} d \lambda \int_{0}^{\infty} d \omega \chi_{m n}^{\lambda}\left(k_{\|}, i \omega\right) \\
& -\frac{\mu_{m} \mu_{n}}{\pi d^{2}} \sum_{l=1}^{l_{M}}\left(E_{F}-\epsilon_{l}\right) \sum_{l^{\prime}=1}^{\infty} G_{l l^{\prime}}^{m} G_{l l^{\prime}}^{n},
\end{aligned}
$$

with $d=a+2 z_{0}$,

$$
\mu_{m}= \begin{cases}1 & \text { for } m=0, \\ 2 & \text { for } m \geqslant 1,\end{cases}
$$

and

$$
G_{l l^{\prime}}^{m}=\frac{1}{2} \sum_{s=1}^{\infty} \sum_{s^{\prime}=1}^{\infty} b_{l s} b_{l^{\prime} s^{\prime}}\left(\delta_{m, s-s^{\prime}}+\delta_{m, s^{\prime}-s}-\delta_{m, s+s^{\prime}}\right) .
$$

${ }^{1}$ W. Kohn and L. J. Sham, Phys. Rev. 140, A1133 (1965).

${ }^{2}$ R. M. Dreizler and E. K. U. Gross, Density-Functional Theory: An Approach to the Quantum Many-Body Problem (Springer, Berlin, 1990).

${ }^{3}$ S. Kurth, J. P. Perdew, and P. Blaha, Int. J. Quantum Chem. 75, 889 (1999)

${ }^{4}$ N. D. Lang and W. Kohn, Phys. Rev. B 1, 4555 (1970).

${ }^{5}$ D. C. Langreth and J. P. Perdew, Phys. Rev. B 15, 2884 (1977); 21, 5469 (1980); 26, 2810 (1982).

${ }^{6}$ J. P. Perdew, K. Burke, and M. Ernzerhof, Phys. Rev. Lett. 77, 3865 (1996), and references therein.

${ }^{7}$ E. Krotscheck, W. Kohn, and G.-X. Qian, Phys. Rev. B 32, 5693 (1985); E. Krotscheck and W. Kohn, Phys. Rev. Lett. 57, 862 (1986).

${ }^{8}$ P. H. Acioli and D. M. Ceperley, Phys. Rev. B 54, 17199 (1996).

${ }^{9}$ W. M. C. Foulkes and B. Wood (private communication).

${ }^{10}$ J. M. Pitarke and J. P. Perdew, Phys. Rev. B 67, 045101 (2003).

${ }^{11}$ J. M. Pitarke, Phys. Rev. B 70, 087401 (2004).

${ }^{12}$ J. M. Pitarke and A. G. Eguiluz, Phys. Rev. B 57, 6329 (1998); 63, 045116 (2001).

${ }^{13}$ Z. Yan, J. P. Perdew, S. Kurth, C. Fiolhais, and L. Almeida, Phys. Rev. B 61, 2595 (2000).

${ }^{14}$ L. M. Almeida, J. P. Perdew, and C. Fiolhais, Phys. Rev. B 66, 075115 (2002).

${ }^{15}$ J. Jung, P. Garcia-González, J. F. Dobson, and R. W. Godby, Phys. Rev. B 70, 205107 (2004).

${ }^{16}$ L. A. Constantin, J. P. Perdew, and J. Tao, Phys. Rev. B 73,
205104 (2006)

${ }^{17}$ J. Tao, J. P. Perdew, V. N. Staroverov, and G. E. Scuseria, Phys. Rev. Lett. 91, 146401 (2003).

${ }^{18}$ J. P. Perdew and Y. Wang, Phys. Rev. B 45, 13244 (1992).

${ }^{19}$ Z. Yan, J. P. Perdew, and S. Kurth, Phys. Rev. B 61, 16430 (2000).

${ }^{20} \mathrm{We}$ are considering here a plane-bounded semi-infinite electron system. In the case of a jellium slab, the right-hand side of Eq. (5) should be divided by a factor of 2 .

${ }^{21}$ J. P. Perdew and Y. Wang, Phys. Rev. B 46, 12947 (1992).

${ }^{22}$ M. Ernzerhof and J. P. Perdew, J. Chem. Phys. 109, 3313 (1998).

${ }^{23}$ D. C. Langreth and J. P. Perdew, Solid State Commun. 17, 1425 (1975).

${ }^{24}$ O. Gunnarsson and B. I. Lundqvist, Phys. Rev. B 13, 4274 (1976).

${ }^{25}$ H. B. Callem and T. R. Welton, Phys. Rev. 83, 34 (1951).

${ }^{26}$ D. Pines and P. Nozieres, The Theory of Quantum Liquids (Addison-Wesley, Reading, MA, 1989).

${ }^{27}$ For the exchange contribution to $\bar{n}_{\mathrm{xc}}^{\text {unif }}(u)$ we have used the nonoscillatory parametrization reported in Ref. 22. For the RPA correlation contribution to $\bar{n}_{\mathrm{xc}}^{\text {unif }}(u)$ we have used the nonoscillatory parametrization reported in Ref. 19 within the framework of Ref. 21.

${ }^{28} z_{0}$ is chosen sufficiently large for the physical results to be insensitive to the precise value employed.

${ }^{29}$ A. G. Eguiluz, Phys. Rev. B 31, 3303 (1985).

${ }^{30}$ D. M. Ceperley and B. J. Alder, Phys. Rev. Lett. 45, 566 (1980). 
${ }^{31}$ M. Rasolt and D. J. W. Geldart, Phys. Rev. B 25, 5133 (1982).

${ }^{32}$ K. Burke and J. P. Perdew, Int. J. Quantum Chem. 56, 199 (1995).

${ }^{33}$ K. Burke, J. P. Perdew, and D. C. Langreth, Phys. Rev. Lett. 73, 1283 (1994).

${ }^{34}$ J. P. Perdew, L. A. Constantin, E. Sagvolden, and K. Burke (unpublished).
${ }^{35}$ TPSS RPA xc surface energies of a semi-infinite jellium are indeed very close to (and usually slightly lower than) their exact RPA counterparts, as shown in Table I of Ref. 16.

${ }^{36}$ J. P. Perdew and K. Schmidt, in Density Functional Theory and Its Application to Materials, edited by V. E. Van Doren, K. Van Alsenoy, and P. Geerlings (AIP, Melville, NY, 2001). 